TRANSACTIONS OF THE

AMERICAN MATHEMATICAL SOCIETY

Volume 349, Number 5, May 1997, Pages 1961-1971

S 0002-9947(97)01796-0

\title{
SINGULAR SET OF SOME KÄHLER ORBIFOLDS
}

\author{
THALIA D. JEFFRES
}

\begin{abstract}
We consider some examples of orbifolds with positive first Chern class. Applying a result of Ding and Tian, we show that the singularities must be very mild if the orbifold admits a Kähler-Einstein metric.
\end{abstract}

\section{BACKGROUND}

A complex manifold is Kähler-Einstein if it admits a metric whose Ricci and Kähler forms are related by $\rho=k \omega$ for a real number $k$. The first Chern class will be positive, negative, or zero according to the sign of $k$. This provides a necessary condition for the existence of a Kähler-Einstein metric. In fact, if $C_{1}(M)=0$ or $C_{1}(M)<0$, this condition is also sufficient, but for $C_{1}(M)>0$ there are examples demonstrating its insufficiency. Additionally, for $C_{1}(M)>0$, obstructions have been found by Matsushima, Futaki, and others. These are discussed in the book by Futaki $([\mathrm{F}])$. Since these automatically vanish when there are no nontrivial holomorphic vector fields, Calabi asked whether a complex manifold with $C_{1}>0$ and no nontrivial holomorphic vector fields must admit a Kähler-Einstein metric. Tian has given an affirmative answer for the surface case ([T]).

Kähler-Einstein metrics can also be defined on many singular varieties, such as Kähler orbifolds with normal singularities. These singularities are of particular interest because they appear in the Hausdorff limit of smooth varieties. For singular surfaces with $C_{1}>0$ new obstructions arise. Indeed, in contrast to the smooth case, there are two-dimensional orbifolds which possess no nontrivial holomorphic vector fields and yet do not admit a Kähler-Einstein metric. More specifically, Ding and Tian proved that a cubic surface in $C P^{3}$ has a Kähler-Einstein metric if and only if it is stable in the sense of Mumford. It seems to be that the correct condition for the existence of a Kähler-Einstein metric is a certain notion of stability in geometric invariant theory.

In this paper we examine a special case. Suppose that $f$ and $g$ are homogeneous quadratic polynomials whose zero locus in $C P^{4}$ is

$$
\Sigma_{f, g}=\left\{\left[z_{0}, \cdots, z_{4}\right] \mid f=g=0\right\} .
$$

Suppose also that $\Sigma_{f, g}$ is an orbifold whose singularities are rational double points. Note that such an orbifold does indeed have positive first Chern class. It is a quartic del Pezzo orbifold. Then the main theorem is as follows:

Main Theorem. If the orbifold described above admits a Kähler-Einstein metric, then it may have only the mildest singularities, those of type $A_{1}$.

Received by the editors November 6, 1995.

1991 Mathematics Subject Classification. Primary 53C55; Secondary 14J17.

(C)1997 American Mathematical Society 
Referring to the work of Mabuchi and Mukai ([MM]), this establishes semistability, giving the link indicated above. It should be noted in passing that in contrast to the work of Mabuchi and Mukai, we do not assume here that the orbifolds are limits of smooth surfaces.

\section{Outline of the Method}

The method may be outlined as follows. Assuming first that a more severe singularity occurs, we use the classification theory of singularities (see for example $[\mathrm{BK}]$ or $[\mathrm{BPV}])$ to recover some information about the form of the original defining polynomials. Typically this orbifold will not admit a nontrivial holomorphic vector field. It may, however, be moved through a one-parameter family of automorphisms of the ambient space to a limit orbifold which does. Computation on this limit of a generalized Futaki invariant developed by Ding and Tian in which the structure of the singularities plays a role can yield information about the original orbifold. Namely, by choosing the automorphism carefully, negativity of this invariant when evaluated on the vector field generated by this one-parameter family implies that the original orbifold does not admit a Kähler-Einstein metric.

This conclusion follows from the main theorem shown by Ding and Tian, which is Theorem 0.2 of their paper $([\mathrm{DT}])$.

Theorem 1 (Ding and Tian). Let $X$ be a Kähler orbifold embedded in $C P^{N}$ such that the hyperplane bundle restricts to a multiple of the anticanonical bundle $K_{X}^{-1}$, and $\sigma_{t}$ a one-parameter subgroup in Aut $\left(C P^{N}\right)=S L(N+1, C)$. Let $V$ be the induced holomorphic vector field. Assume that the $X_{t}=\sigma_{t}(X)$ converge to an irreducible, normal, and nondegenerate variety $X_{\infty}$ and that $X_{\infty}$ admits a KählerEinstein orbifold metric. Then the generalized Futaki invariant computed on $X_{\infty}$, $F_{\infty}(V)$, is nonnegative.

For the purposes of the computations in this paper, there is the following formula for the Futaki invariant, also established by Ding and Tian. Before stating this, we first establish some notations. Let $\Lambda=\Lambda_{0} \cup \Lambda_{1}$ be the fixed point set of the one-parameter family of automorphisms $\left\{\sigma_{t}\right\}$, where $\Lambda_{i}$ consists in the components with dimension $i$. Let $N_{\lambda}$ denote the normal bundle of the component $Z_{\lambda} \subset \Lambda$ in $\sigma$. Define a map $L(V): N_{\lambda} \longrightarrow N_{\lambda}$ by $L(V) Y=\left(D_{V} Y\right)^{\perp}$, where $D$ denotes the connection. Suppose that $L(V)$ is nondegenerate, that is, that $\operatorname{det}(L(V))$ is nonzero.

Theorem 2 (Ding and Tian). For complex dimension two,

$$
\begin{aligned}
F_{X}(V) & =\sum_{\Lambda_{0}} \frac{1}{\left|\Gamma_{x}\right|} \frac{(\operatorname{div} V)^{3}}{\operatorname{det} L(V)} \\
& +\sum_{\Lambda_{1}} \operatorname{div}_{C}(V)\left(2 \operatorname{deg}(C)+\frac{1}{|\Gamma|}\left(2-2 g(C)-\sum_{x \in C} \frac{\left|\Gamma_{x}\right|-1}{\left|\Gamma_{x}\right|}\right)\right)
\end{aligned}
$$

Here, $g(C)$ denotes the genus of the one-dimensional component $C .\left|\Gamma_{x}\right|$ is the order of the uniformization group at the point $x$, and $|\Gamma|$ is the order of the local uniformization group at a generic point on $C . \operatorname{div}_{C}(V)$ is the normal component of the divergence. 


\section{A Formula for $A_{n}$ Singularities}

A surface $\Sigma$ with a rational double point may be expressed locally as a hypersurface in $C^{3}$. At the same time, the singularity is the quotient $D^{2} / G$, where $G$ is a finite subgroup of $S U(2)$. Now if $X$ is a vector field on all of $C^{3}$, it is useful to develop a formula which allows one to compute the contribution of this point to the Futaki invariant directly, without having first to parametrize the surface and compute the local lift $X_{0}$ of $X$ to the disk $D^{2}$. The most commonly occurring singularities on these surfaces are the $A_{k}$ singularities, and it is easy in this case.

Locally, such a singularity is described by $z_{0} z_{1}+z_{2}^{n}$. There is a map from the disk to the singular surface,

$$
(u, v) \rightarrow\left(u^{n}, v^{n}, \varepsilon u v\right),
$$

where $\varepsilon$ is any complex number with $\varepsilon^{n}=-1$. The vector field $X$ is generated by a one-parameter family of automorphisms

$$
\sigma(t):\left(z_{0}, z_{1}, z_{2}\right) \rightarrow\left(t^{k_{0}} z_{0}, t^{k_{1}} z_{1}, t^{k_{2}} z_{2}\right),
$$

so that

$$
X=\sum_{0}^{2} k_{i} z_{i} \frac{\partial}{\partial z_{i}}
$$

If $X_{0}$ is a local lifting of $X$ to the disk $D^{2}$, in other words, $\pi_{*} X_{0}=X$, then one computes that

$$
X_{0}=\frac{1}{n} k_{0} u \frac{\partial}{\partial u}+\frac{1}{n} k_{1} v \frac{\partial}{\partial v}
$$

so that

$$
\operatorname{div} X_{0}=\frac{1}{n}\left(k_{0}+k_{1}\right)=k_{2} .
$$

$\frac{1}{n}\left(k_{0}+k_{1}\right)=k_{2}$ holds because the one-parameter family of automorphisms leaves the surface invariant. Therefore the contribution to the Futaki invariant of this point is

$$
\frac{\left(\operatorname{div} X_{0}\right)^{3}}{|G| \operatorname{det} X_{0}}=\frac{n k_{2}^{3}}{k_{0} k_{1}}
$$

\section{Proof of the Main Result}

Since $\Sigma_{f, g}$ is not smooth, we may assume after a change of variables that a singularity occurs at the point $[1,0,0,0,0]$. Then after a further change of variables, we may assume that $f$ and $g$ are of the form

$$
\begin{aligned}
& f=z_{0} z_{1}+f_{2}\left(z_{2}, z_{3}, z_{4}\right), \\
& g=g_{2}\left(z_{1}, z_{2}, z_{3}, z_{4}\right) .
\end{aligned}
$$

For the proof, we consider different cases, depending on the rank of $f_{2}$. The lowest rank case is the simplest, for one has:

Lemma 1. If the rank of $f_{2}$ is one, then $\Sigma_{f, g}$ may have only singularities of type $A_{k}$. 
Proof of the lemma. Assume as before that the singularity occurs at $[1,0,0,0,0]$. Then if $f_{2}$ has rank one, $f$ and $g$ may be expressed as

$$
\begin{aligned}
& f=z_{0} z_{1}+z_{2}^{2}, \\
& g=z_{1} \sum_{1}^{4} a_{i} z_{i}+Q\left(z_{2}, z_{3}, z_{4}\right) .
\end{aligned}
$$

Showing that only $A_{k}$ singularities occur is the same as showing that $Q$ has rank at least two, because the singularity is described locally in $U_{0}$ by

$$
G^{*}=a_{1} z_{2}^{4}-z_{2}^{2} \sum_{1}^{4} a_{i} z_{i}+Q\left(z_{2}, z_{3}, z_{4}\right),
$$

and so

$$
\frac{\partial^{2} G^{*}}{\partial z_{i} \partial z_{j}}(0,0,0)=\frac{\partial^{2} Q}{\partial z_{i} \partial z_{j}}(0,0,0)
$$

If $Q$ has rank less than two, it must have rank one, since $g$ is irreducible, and so $Q$ can be written as $Q\left(z_{2}, z_{3}, z_{4}\right)=\left(a z_{2}+b z_{3}+c z_{4}\right)^{2}$. If $b$ and $c$ are both zero, then $g$ is either reducible or degenerate, so we may assume this does not happen; therefore assume without loss of generality that it is $b$ which is nonzero. Then after a change of variables, $f$ and $g$ may be written as

$$
\begin{aligned}
& f=z_{0} z_{1}+z_{2}^{2}, \\
& g=z_{1} \sum_{1}^{4} a_{i} z_{i}+z_{3}^{2},
\end{aligned}
$$

where $a_{4}$ cannot be zero or again $\Sigma_{f, g}$ will be degenerate. Then the further change of variables $w_{4}=\sum_{1}^{4} a_{i} z_{i}$ and $w_{i}=z_{i}$ for $i \neq 4$ expresses $f$ and $g$ as

$$
\begin{aligned}
& f=w_{0} w_{1}+w_{2}^{2}, \\
& g=w_{1} w_{4}+w_{3}^{2},
\end{aligned}
$$

showing that this singularity is nonsimple. Therefore the rank of $Q$ must be at least 2 .

We now proceed to the proof of the main theorem.

Case 1. The rank of $f_{2}$ is 1 .

While the above shows that $Q$ must have rank at least two, if we would like to assume $\Sigma_{f, g}$ has a singularity worse that $A_{1}$ at $[1,0,0,0,0]$ then the rank of $Q$ cannot exceed two either. If it did, then

$$
G^{*}=a_{1} z_{2}^{4}-z_{2}^{2} \sum_{2}^{4} a_{i} z_{i}+Q\left(z_{2}, z_{3}, z_{4}\right)
$$

could be diagonalized as

$$
G^{*}=w^{2}+x^{2}+z^{2}
$$


which describes an $A_{1}$ singularity. So we have

$$
\begin{aligned}
& f=z_{0} z_{1}+z_{2}^{2}, \\
& g=z_{1} \sum_{1}^{4} a_{i} z_{i}+Q\left(z_{2}, z_{3}, z_{4}\right),
\end{aligned}
$$

with $\operatorname{rank} Q=2$.

After a linear change of variables, these may be rewritten as

$$
\begin{aligned}
& f=z_{0} z_{1}+f_{2}(w, x, z), \\
& g=a_{1} z_{1}^{2}+z_{1} \ell(w, x, z)+w^{2}+x^{2},
\end{aligned}
$$

where $\ell$ denotes a linear function. $f_{2}(w, x, z)$ still has rank 1 , so

$$
f_{2}(w, x, z)=(a w+b x+c z)^{2} .
$$

One checks that $c$ cannot be zero, or else $\Sigma_{f, g}$ has nonisolated singularities. This allows one to redefine $z$ as $a w+b x+c z$ and rewrite $f$ as $f=z_{0} z_{1}+z^{2}$. Completing the square on the $w$ and $x$ terms in $g$ brings $f$ and $g$ to the form

$$
\begin{aligned}
& f=z_{0} z_{1}+z^{2}, \\
& g=a_{1} z_{1}^{2}+a_{2} z_{1} z+w^{2}+x^{2} .
\end{aligned}
$$

If $a_{2} \neq 0$, then putting $\tilde{z}=a_{1} z_{1}+a_{2} z$, redefining $z_{0}$ and absorbing the constants brings $f$ and $g$ to the form

$$
\begin{aligned}
& f=z_{0} z_{1}+z^{2}, \\
& g=z_{1} z+w^{2}+x^{2} .
\end{aligned}
$$

If $a_{2}=0$, then $f$ and $g$ may be brought to the form

$$
\begin{aligned}
& f=z_{0} z_{1}+z^{2}, \\
& g=z_{1}^{2}+w^{2}+x^{2} .
\end{aligned}
$$

In either case, $\Sigma_{f, g}$ already admits a nontrivial holomorphic vector field, and one computes $F(X) \neq 0$, showing that neither of these surfaces admits a KählerEinstein metric.

Case 2. The rank of $f_{2}$ is 2 .

Now suppose the rank of $f_{2}$ is 2 , so that $f$ and $g$ initially look like

$$
\begin{aligned}
& f=z_{0} z_{1}+z_{2}^{2}+z_{3}^{2}, \\
& g=z_{1} \sum_{1}^{4} a_{i} z_{i}+Q\left(z_{2}, z_{3}, z_{4}\right) .
\end{aligned}
$$

First suppose that $Q$ has rank 1 . Then $g$ may be written

$$
g=z_{1} \sum_{1}^{4} a_{i} z_{i}+\left(a z_{2}+b z_{3}+c z_{4}\right)^{2}
$$

It is not necessary to consider the case in which $c=0$. To see this, first ask whether it is possible to replace $f$ by $f+\lambda g$ of smaller rank:

$$
f+\lambda g=z_{1}\left(z_{0}+\sum_{1}^{4} \lambda a_{i} z_{i}\right)+z_{2}^{2}+z_{3}^{2}+\lambda\left(a z_{2}+b z_{3}\right)^{2} .
$$


Define

$$
R_{\lambda}\left(z_{2}, z_{3}\right)=z_{2}^{2}+z_{3}^{2}+\lambda\left(a z_{2}+b z_{3}\right)^{2}
$$

Then

$$
\operatorname{det}(\lambda):=\operatorname{det} \frac{\partial^{2} R_{\lambda}}{\partial z_{2} \partial z_{3}}=4\left(1+\left(a^{2}+b^{2}\right) \lambda\right),
$$

so that if $a^{2}+b^{2} \neq 0$, then it is possible to solve $\operatorname{det}(\lambda)=0$, reducing the rank. We do not wish to study this, as it is included in Case 1, and so we may assume $a^{2}+b^{2}=0$. Normalizing so that $a=1$ and supposing without loss of generality that $b=+i$, we get

$$
\begin{aligned}
& f=z_{0} z_{1}+\left(z_{2}+i z_{3}\right)\left(z_{2}-i z_{3}\right), \\
& g=z_{1} \sum_{1}^{4} a_{i} z_{i}+\left(z_{2}+i z_{3}\right)^{2} .
\end{aligned}
$$

After the obvious change of variables, this becomes

$$
\begin{aligned}
& f=z_{0} z_{1}+w x \\
& g=z_{1}\left(a_{1} z_{1}+a_{2}^{\prime} w+a_{3}^{\prime} x+a_{4} z_{4}\right)+w^{2} .
\end{aligned}
$$

$a_{4}$ must be nonzero or else $\Sigma_{f, g}$ is degenerate, so putting $z=a_{1} z_{1}+a_{2}^{\prime} w+a_{3}^{\prime} x+a_{4} z_{4}$ gives

$$
\begin{aligned}
& f=z_{0} z_{1}+w x, \\
& g=z_{1} z+w^{2} .
\end{aligned}
$$

From this form it is easy to recognize that $\Sigma_{f, g}$ has nonisolated singularities. Therefore we may assume in the original expression for $Q$ that $c \neq 0$. This allows us to simplify the form of $f$ and $g$ and finally write

$$
\begin{aligned}
& f=z_{0} z_{1}+w^{2}+x^{2}, \\
& g=a_{1} z_{1}^{2}+z_{1} \ell(w, x)+z^{2} .
\end{aligned}
$$

Note that unlike the earlier example, here $\Sigma_{f, g}$ does not admit a nontrivial holomorphic vector field. We want to choose a 1-parameter family of automorphisims in such a way that

1. $\Sigma_{f, g} \stackrel{\sigma(t)}{\longrightarrow} \Sigma_{\infty}$ with $\Sigma_{\infty}$ irreducible and nondegenerate,

2. $\Sigma_{\infty}$ is invariant under $\sigma(t)$, so that $\sigma^{\prime}(1)$ is a nontrivial holomorphic vector field on $\Sigma_{\infty}$, and

3. $F_{\infty}\left(\sigma^{\prime}(1)\right)<0$.

This will show, by the theorem of Ding and Tian, that the original $\Sigma_{f, g}$ cannot admit a Kähler-Einstein metric.

If we write

$$
\sigma(t):\left[z_{0}, \cdots, z_{4}\right] \mapsto\left[z_{0}, t^{\alpha_{1}} z_{1}, \cdots, t^{\alpha_{4}} z_{4}\right],
$$

consideration of the three requirements above already determines the weights $\alpha_{i}$. For instance, since $g$ may have few terms, and we want $\Sigma_{\infty}$ to be irreducible, try to choose $\sigma(t)$ so that $f$ is invariant. Then $\sigma(t)$ must have the form

$$
\sigma(t):\left[z_{0}, z_{1}, w, x, z\right] \mapsto\left[z_{0}, t^{2 \alpha} z_{1}, t^{\alpha} w, t^{\alpha} x, t^{\beta} z\right] .
$$


Polynomials $f_{t}$ and $g_{t}$ are determined by

$$
\sigma(t)\left(\Sigma_{f, g}\right)=\Sigma_{f_{t}, g_{t}},
$$

in other words, the image $\sigma(t)\left(\Sigma_{f, g}\right)$ is the zero locus of $f_{t}$ and $g_{t}$. One computes

$$
g_{t}=a_{1} t^{2 \beta-4 \alpha} z_{1}^{2}+t^{2 \beta-3 \alpha} z_{1} \ell(w, x)+z^{2} .
$$

$\ell$ is not zero since the original surface was irreducible. So for $g_{t}$ to converge, $\beta$ must be $\beta=\frac{3}{2} \alpha$. Then $\Sigma_{f, g}$ converges to a limit orbifold $\Sigma_{\infty}$ described by

$$
\begin{aligned}
& f_{\infty}=z_{0} z_{1}+w^{2}+x^{2}, \\
& g_{\infty}=z_{1} \ell(w, x)+z^{2}
\end{aligned}
$$

via $\sigma(t):\left[z_{0}, z_{1}, w, x, z\right] \mapsto\left[z_{0}, t^{2 \alpha} z_{1}, t^{\alpha} w, t^{\alpha} x, t^{\frac{3}{2}} \alpha\right]$.

Writing $f_{2}$ as $f_{2}=w^{2}+x^{2}$ was useful to fix the weights and determine $\sigma(t)$, but to compute the Futaki invariant it is easier now to write this as $f_{2}=w x$ so that $f=z_{0} z_{1}+w x$; since $w$ and $x$ have the same weights, the form of $\sigma(t)$ is not affected.

The fixed point set of $\sigma(t)$ is

$$
\Lambda(x)=\{[1,0,0,0,0],[0,1,0,0,0],[0,0,1,0,0],[0,0,0,0,1]\} .
$$

The first fixed point is a $D_{4}$ singularity and the others are smooth. One computes

$$
F_{\infty}\left(\sigma^{\prime}(1)\right)=\frac{1}{4} \alpha-\frac{27}{4} \alpha+\frac{1}{4} \alpha+\frac{1}{4} \alpha<0 .
$$

Now suppose that rank $Q=2$. Then there is a linear change of variables so that

$$
\begin{aligned}
& f=z_{0} z_{1}+f_{2}(w, x, z), \\
& g=a_{1} z_{1}^{2}+z_{1} \ell(w, x, z)+w^{2}+x^{2} .
\end{aligned}
$$

$f_{2}$ still has rank two, and it must contain $z$, for if it does not, it is possible to reduce the rank by replacing $f$ with $f+\lambda g$.

Next, consider two cases, depending on the form of $\ell(w, x, z)$.

I. Suppose that $\ell(w, x, z)=a w+b x+c z$, with $c \neq 0$. Then after a change of variables, $f$ and $g$ may be written in the form

$$
\begin{aligned}
& f=z_{0} z_{1}+f_{2}(w, x, z), \\
& g=z_{1} z+w^{2}+x^{2} .
\end{aligned}
$$

Rewriting $f$ in more detail indicates how to choose $\sigma(t)$ and $\Sigma_{\infty}$ :

$$
\begin{aligned}
& f=z_{0} z_{1}+A z^{2}+z \ell(w, x)+Q(w, x), \\
& g=z_{1} z+w^{2}+x^{2} .
\end{aligned}
$$

Here, $A$ or $\ell$ can be zero, but they cannot both vanish.

If $A \neq 0$, choose

$$
\sigma(t):\left[z_{0}, z_{1}, w, x, z\right] \mapsto\left[z_{0}, t^{\frac{4}{3} \alpha} z_{1}, t^{\alpha} w, t^{\alpha} x, t^{\frac{2}{3} \alpha} z\right] .
$$

The same computation as before shows that $F_{\infty}\left(\sigma^{\prime}(1)\right)<0$.

If $A=0$, then choose

$$
\sigma(t):\left[z_{0}, z_{1}, w, x, z\right] \mapsto\left[z_{0}, t^{3 \alpha} z_{1}, t^{2 \alpha} w, t^{2 \alpha} x, t^{\alpha} z\right]
$$


and converge to

$$
\begin{aligned}
& f_{\infty}=z_{0} z_{1}+z \ell(w, x), \\
& g_{\infty}=z_{1} z+w^{2}+x^{2} .
\end{aligned}
$$

Again, to compute $F_{\infty}\left(\sigma^{\prime}(1)\right)$ it is more convenient to rewrite these as

$$
\begin{aligned}
& f_{\infty}=z_{0} z_{1}+z \hat{\ell}(w, x), \\
& g_{\infty}=z_{1} z+w x,
\end{aligned}
$$

and it is also important to notice that $\hat{\ell}$ must contain both $w$ and $x$ or else the original surface $\Sigma_{f, g}$ has nonisolated singularities. Then one computes $F_{\infty}\left(\sigma^{\prime}(1)\right)<$ 0 .

II. Now suppose $\ell(w, x, z)$ does not contain $z$, in other words that

$$
\begin{aligned}
& f=z_{0} z_{1}+f_{2}(w, x, z), \\
& g=a_{1} z_{1}^{2}+z_{1} \ell(w, x)+w^{2}+x^{2} .
\end{aligned}
$$

By completing the square in $g$ on $w$ and $x$, and rescaling, one may assume

$$
\begin{aligned}
& f=z_{0} z_{1}+f_{2}(w, x, z), \\
& g=z_{1}^{2}+w^{2}+x^{2} .
\end{aligned}
$$

Rewriting $f$ in more detail as

$$
f=z_{0} z_{1}+a z^{2}+z \ell(w, x)+Q(w, x)
$$

one checks that if $A=0$, then $\Sigma_{f, g}$ has nonisolated singularities.

Choose $\sigma(t):\left[z_{0}, z_{2}, w, x, z\right] \mapsto\left[z_{0}, t^{2 \alpha} z_{1}, t^{2 \alpha} w, t^{2 \alpha} x, t^{\alpha} z\right], \Sigma_{f, g} \stackrel{\sigma(t)}{\longrightarrow} \Sigma_{\infty}$, described by

$$
\begin{aligned}
& f_{\infty}=z_{0} z_{1}+z^{2} \\
& g_{\infty}=z_{1}^{2}+w^{2}+x^{2},
\end{aligned}
$$

or, equivalently,

$$
\begin{aligned}
& f_{\infty}=z_{0} z_{1}+z^{2} \\
& g_{\infty}=z_{1}^{2}+w x
\end{aligned}
$$

The fixed point set of $\sigma(t)$ is

$$
\Lambda(X)=\{[1,0,0,0,0]\} \cup\left\{\left[0, z_{1}, w, x, 0\right] \mid z_{1}^{2}+w x=0\right\} .
$$

$[1,0,0,0,0]$ is an $A_{3}$ singularity, and contributes $\alpha$ to the Futaki invariant. The contribution of the 1-dimensional component, $C$, is

$$
\begin{aligned}
\operatorname{div}_{C} x(2 \cdot \operatorname{deg} C & \left.+\frac{1}{|\Gamma|}\left(2-2 g(C)-\sum_{x \in C} \frac{\left|\Gamma_{x}\right|-1}{\left|\Gamma_{x}\right|}\right)\right) \\
& =-\alpha\left(2 \cdot 2+1\left(2-0-\left(\frac{1}{2}+\frac{1}{2}\right)\right)\right),
\end{aligned}
$$

so $F_{\infty}\left(\sigma^{\prime}(1)\right)=\alpha-15 \alpha<0$. This completes the second case, when $f_{2}$ has rank 2 .

Case 3. $f$ has full rank. 
In this case, $f$ and $g$ may be written as

$$
\begin{aligned}
& f=z_{0} z_{1}+z_{2}^{2}+z_{3}^{2}+z_{4}^{2}, \\
& g=z_{1} \sum_{1}^{4} a_{i} z_{i}+Q\left(z_{2}, z_{3}, z_{4}\right) .
\end{aligned}
$$

We consider separately the cases $\operatorname{rank} Q=1$ and $\operatorname{rank} Q=2$, beginning with the former. In this instance, $f$ and $g$ may be written, after a change of variables, as

$$
\begin{aligned}
& f=z_{0} z_{1}+f_{2}(w, x, z), \\
& g=z_{1} w+x^{2} .
\end{aligned}
$$

$f_{2}(w, x, z)$ is still homogeneous, quadratic, and has rank 3 . Furthermore, $f_{2}$ must contain the term $z^{2}$ or else the surface will have nonisolated singularities, so $f$ and $g$ are of the form

$$
\begin{aligned}
& f=z_{0} z_{1}+Q(w, x)+z^{2}, \\
& g=z_{1} w+x^{2} .
\end{aligned}
$$

To find $\sigma(t)$ and $\Sigma_{\infty}$, we reason this way; we must have $g_{\infty}=g$, and we also want to keep $z^{2}$ in $f_{\infty}$. This partially determines the weights;

$$
\sigma(t):\left[z_{0}, z_{1}, w, x, z\right] \mapsto\left[z_{0}, t^{2 \alpha} z_{1}, t^{2 \beta-2 \alpha} w, t^{\beta} x, t^{\alpha} z\right]
$$

with $2 \alpha+\beta=2 \gamma$. Then compute that $\sigma(t)\left(\Sigma_{f, g}\right)$ is the zero locus of $g$ and of

$$
f_{t}=z_{0} z_{1}+a t^{6 \alpha-4 \beta} w^{2}+b t^{4 \alpha-3 \beta} w x+c t^{2 \alpha-2 \beta} x^{2}+z^{2} .
$$

In order for $f_{t}$ to converge as $t \rightarrow \infty$, there are the following conditions:

$$
\begin{aligned}
& a \neq 0 \quad \Rightarrow \quad \alpha \leq \frac{2}{3} \beta, \\
& b \neq 0 \quad \Rightarrow \quad \alpha \leq \frac{3}{4} \beta, \\
& c \neq 0 \quad \Rightarrow \quad \alpha \leq \beta .
\end{aligned}
$$

So if $a$ is nonzero, we must choose $\alpha=\frac{2}{3} \beta$. If $a=0$, we must choose $\alpha=\frac{3}{4} \beta$, for in this case $b$ cannot be zero.

In the former possibility, then, $\Sigma_{f, g}$ converges to a limit orbifold described by

$$
\begin{aligned}
& f_{\infty}=z_{0} z_{1}+w^{2}+z^{2}, \\
& g_{\infty}=z_{1} w+x^{2}
\end{aligned}
$$

via $\sigma(t):\left[z_{0}, z_{1}, w, x, z\right] \mapsto\left[z_{0}, t^{\frac{4}{3} \beta} z_{1}, t^{\frac{2}{3} \beta} w, t^{\beta} x, t^{\frac{2}{3} \beta} z\right]$. An earlier computation shows that

$$
F_{\infty}\left(\sigma^{\prime}(1)\right)<0
$$

In the latter possibility, we have

$$
\begin{aligned}
& f_{\infty}=z_{0} z_{1}+w x+z^{2}, \\
& g_{\infty}=z_{1} w+x^{2},
\end{aligned}
$$

via $\sigma(t):\left[z_{0}, z_{1}, w, x, z\right] \mapsto\left[z_{0}, t^{6 \beta} z_{1}, t^{2 \beta} w, t^{4 \beta} x, t^{3 \beta} z\right]$. The fixed point set is

$$
\Lambda(X)=\{[1,0,0,0,0],[0,1,0,0,0],[0,0,1,0,0]\} .
$$


It is interesting that among the limit orbifolds which arise in this context, this one has the highest order singularity. $[1,0,0,0,0]$ is a $D_{5}$ singularity; the others are smooth points. One computes $F_{\infty}\left(\sigma^{\prime}(1)\right)=\frac{\beta}{3}-\frac{5^{3}}{6} \beta+\frac{1}{2} \beta<0$.

It remains now to examine what happens when $\operatorname{rank} Q=2$. A linear change of variables diagonalizes $Q$ :

$$
\begin{aligned}
& f=z_{0} z_{1}+f_{2}(w, x, z), \\
& g=a_{1} z_{1}^{2}+z_{1} \ell(w, x, z)+w^{2}+x^{2} .
\end{aligned}
$$

Write $f_{2}=a z^{2}+z \ell(w, x)+Q(w, x)$. If $a \neq 0$, normalize so that $a=1$ and complete the square so that $f$ and $g$ now have the form

$$
\begin{aligned}
& f=z_{0} z_{1}+Q(w, x)+z^{2}, \\
& g=a_{1} z_{1}^{2}+z_{1} \ell(w, x, z)+w^{2}+x^{2} .
\end{aligned}
$$

To keep $\Sigma_{\infty}$ as uncomplicated as possible and to avoid converging to something possibly irreducible or degenerate, try to keep $w^{2}, x^{2}$, and $z^{2} ; \sigma(t)$ must then look like

$$
\sigma(t):\left[z_{0}, z_{1}, w, x, z\right] \mapsto\left[z_{0}, t^{2 \alpha} z_{1}, t^{\beta} w, t^{\beta} x, t^{\alpha} z\right] .
$$

Then $\sigma(t)\left(\Sigma_{f, g}\right)$ is the zero locus of

$$
\begin{aligned}
& f_{t}=z_{0} z_{1}+t^{2 \alpha-2 \beta} Q(w, x)+z^{2}, \\
& g_{t}=a_{1} t^{2 \beta-4 \alpha} z_{1}^{2}+t^{\beta-2 \alpha} z_{1} \ell(w, x)+c t^{2 \beta-3 \alpha} z_{1} z+w^{2}+x^{2} .
\end{aligned}
$$

If $c \neq 0$, we must choose $\beta=\frac{3}{2} \alpha$, and then obtain

$$
\begin{aligned}
& f_{\infty}=z_{0} z_{1}+z^{2}, \\
& g_{\infty}=z_{1} z+w^{2}+x^{2}
\end{aligned}
$$

via $\sigma(t):\left[z_{0}, z_{1}, w, x, z\right] \mapsto\left[z_{0}, t^{2 \alpha} z_{1}, t^{\frac{3}{2} \alpha} w, t^{\frac{3}{2} \alpha} x, t^{\alpha} z\right]$. If $c=0$, choose $\beta=2 \alpha$ and then obtain, after completing the square,

$$
\begin{aligned}
& f_{\infty}=z_{0} z_{1}+z^{2}, \\
& g_{\infty}=z_{1}^{2}+w^{2}+x^{2} .
\end{aligned}
$$

For both of these possibilities, we already computed $F_{\infty}\left(\sigma^{\prime}(1)\right)<0$.

We now must consider the case $a=0$, i.e., $f_{2}$ does not contain $z^{2}$.

After a change of variables, $f$ and $g$ may be written as either

$$
\begin{aligned}
& f=z_{0} z_{1}+z \ell(w, x)+Q(w, x), \\
& g=z_{1}^{2}+w x
\end{aligned}
$$

or

$$
g=z_{1} z+w x .
$$

But the first possibility for $g$ may be eliminated because this surface has nonisolated singularities. In the remaining case, there are two further cases. If $\ell(w, x)=a w+b x$ with $a$ and $b$ both nonzero, then $\Sigma_{f, g}$ can be moved to $\Sigma_{\infty}$ with

$$
\begin{aligned}
& f_{\infty}=z_{0} z_{1}+z \ell(w, x), \\
& g_{\infty}=z_{1} z+w x
\end{aligned}
$$

via $\sigma(t):\left[z_{0}, z_{1}, w, x, z\right] \mapsto\left[z_{0}, t^{3 \alpha} z_{1}, t^{2 \alpha} w, t^{2 \alpha} x, t^{\alpha} z\right]$, for which we already know $F_{\infty}\left(\sigma^{\prime}(1)\right)<0$ from before. If, however, one of $a$ or $b$ is zero, then this choice of 
$\sigma(t)$ produces a limit $\Sigma_{\infty}$ with nonisolated singularities. Suppose without loss of generality that $b=0$, so that $f$ and $g$ look like

$$
\begin{aligned}
& f=z_{0} z_{1}+z w+Q(w, x), \\
& g=z_{1} z+w x .
\end{aligned}
$$

Since $f_{2}$ has rank $3, Q$ must contain $x^{2}$, so $f=z_{0} z_{1}+z w+A w^{2}+B w x+C x^{2}, C \neq 0$. Try to converge to $f_{\infty}=z_{0} z_{1}+z w+C x^{2}$; indeed, this establishes the weights as

$$
\sigma(t):\left[z_{0}, z_{1}, w, x, z\right] \mapsto\left[z_{0}, t^{2 \alpha} z_{1}, t^{\frac{3}{2} \alpha} w, t^{\alpha} x, t^{\frac{1}{2} \alpha} z\right],
$$

and

$$
\begin{aligned}
& f_{\infty}=z_{0} z_{1}+z w+C x^{2}, \\
& g_{\infty}=z_{1} z+w x .
\end{aligned}
$$

This surface has one singularity, of type $A_{4}$ :

$$
\begin{gathered}
\Lambda(X)=\{[1,0,0,0,0],[0,1,0,0,0],[0,0,1,0,0],[0,0,0,0,1]\}, \\
F_{\infty}\left(\sigma^{\prime}(1)\right)=\frac{5}{12} \alpha-\frac{27}{4} \alpha+\frac{4}{3} \alpha+0<0 .
\end{gathered}
$$

This completes the last case, and with it, the proof of the main result.

\section{REFERENCES}

[BK] D. Bättig and H. Knörrer, "Singularitäten", Birkhäuser, 1991.

[BPV] W. Barth, C. Peters, and A. van de Ven, Compact Complex Surfaces, Springer-Verlag, 1984. MR 86c: 32026

[DT] W. Ding and G. Tian, Kaehler-Einstein metrics and the generalized Futaki invariant, Invent Math. 110 (1992), 315-335. MR 93m:53039

[F] A. Futaki, Kaehler-Einstein metrics and integral invariants, Lecture Notes in Math., vol. 13, 14, Springer-Verlag, 1988. MR 90a:53053

[MM] T. Mabuchi and S. Mukai, Stability and Einstein-Kaehler metric of a quartic del Pezzo surface, Einstein Metrics and Yang-Mills Connections (Proc. 27th Taniguchi Internat. Sympos., Savda, 1990; T. Mabuchi and S. Mukai, editors), Lecture Notes in Pure Appl. Math., vol. 145, Marcel Dekker, New York, 1993, pp. 133-160. MR 94m:32043

[T] G. Tian, On Calabi's conjecture for complex surfaces with positive first Chern class, Invent Math. 101 (1991), 101-172. MR 91d:32042

Department of Mathematics, State University of New York, Stony Brook, New York 11794-3651

Current address: Department of Mathematics, University of California at Irvine, Irvine, California 92697-3875

E-mail address: tjeffres@math.uci.edu 